\title{
Improving access to and effectiveness of mental health care for personality disorders: the guideline-informed treatment for personality disorders (GIT-PD) initiative in the Netherlands
}

\author{
Joost Hutsebaut ${ }^{1,2^{*}}$ (D) Ellen Willemsen ${ }^{2,3}$, Nathan Bachrach ${ }^{4,5,6}$ and Rien Van ${ }^{7}$
}

\begin{abstract}
Evidence-based treatment for patients suffering from personality disorders (PDs) is only available to a limited extend in the Netherlands. Consequently, most patients receive non-manualized, unspecialized care.

This manuscript describes the background, rationale and design of the Guideline-Informed Treatment for Personality Disorders (GIT-PD) initiative. GIT-PD aims to provide a simple, principle-driven, 'common-factors' framework for the treatment of PDs. The GIT-PD framework integrates scientific knowledge, professional expertise and patient experience to design a good-enough practice, based on common factors. It offers a basic framework including general principles, a structured clinical pathway, a basic professional stance, interventions focused on common factors, and team and organizational strategies, based on common features of evidence-based treatments and generic competences of professionals. The GIT-PD initiative has had a large impact on the organization of treatment for PDs in the Netherlands. For countries with an interest in improving their health care system for PDs, it could serve as a template that requires only limited resources.
\end{abstract}

Keywords: Personality disorders, Generalist treatment, Implementation, Dissemination, Common factors

\section{Background}

Personality disorders (PDs) are a highly prevalent mental condition world-wide, associated with lifelong social and professional disability [1,2], reduced life expectancy [3], and high societal and health care costs [4]. Following a range of studies showing effectiveness of psychotherapy for PD patients [5], there now is a rather optimistic view of the treatability of PDs. Several national guidelines recommend one of the evidence-based psychotherapy programs, such as Dialectical Behavior Therapy, Schema

\footnotetext{
* Correspondence: Joost.Hutsebaut@deviersprong.nl

${ }^{1}$ Viersprong Institute for Studies on Personality Disorders, Halsteren, The Netherlands

${ }^{2}$ Centre of Expertise on Personality Disorders, Utrecht, The Netherlands Full list of author information is available at the end of the article
}

Therapy and Mentalization-Based Treatment [6-8]. However, implementation of these guidelines in clinical practice is cumbersome and most PD patients do not receive psychotherapy [9]. This is mainly due to capacity problems: delivering specialist psychotherapy is expensive and there is a lack of sufficiently trained professionals due to long training trajectories $[10,11]$. Various studies also indicate that the sustainability of highly specialized evidence-based psychotherapy programs both for PD and for other complex disorders in the mental health services is often not guaranteed [12-14]. As a consequence, even in a country like the Netherlands, with relatively accessible mental health care services, only $23 \%$ of Borderline PD (BPD) patients received some sort of psychotherapy, not necessarily evidence-based, 
and usually in a lower dosage than recommended by the guidelines [10]. The large majority of PD patients therefore receives non-manualized "treatment as usual", of which the content, duration, dosage and setting can vary widely. This limits the overall effectiveness of PD treatments, which may be reflected in the ongoing high consumption of different sorts of care by PD patients [15]. From a health economics point of view, improving access to psychotherapeutically informed care for PD patients may be a cost-effective strategy. The annual cost of PDs in Europe is estimated as in excess of $€ 25$ billion [16] and several studies have demonstrated costeffectiveness of psychotherapies for PDs $[4,17,18]$.

In order to provide an accessible and feasible service for PDs, the Guideline-Informed Treatment for Personality Disorders (GIT-PD) project was started in the Netherlands. The project has realized a significant reform of health care services for PDs, providing an evidenceinformed framework based on common features of specialized psychotherapies for treatment of PDs. Compared to the theory driven approach of specialist treatments, GIT-PD takes a rather pragmatic approach, focusing on common principles and stressing the organizational requirements for delivering good care for PD patients. In addition, GIT-PD requires less training and it upgrades existing services rather than that it completely reforms them, which may add to a simpler implementation. In this paper we present the general design and focus on organizational requirements and strategies for a successful implementation of this service-reform.

\section{Rationale of GIT-PD}

Despite differences in theoretical background and approach, all specialist psychotherapy programs seem to be roughly equally effective [19]. According to Weinberg and colleagues, this may point to the importance of common features across the evidence-based approaches for PD rather than their specific features [20]. Based upon an inspection of the respective manuals, these authors identified five features that may explain the similar effects: clearly structured treatment framework, active stance of the therapist, focus on therapist-patient relationship, the use of interventions aimed at exploring the relationship between emotions, thoughts and behavior, and interventions to support motivation for change. These common features may facilitate the workings of common factors in the treatment of PDs, including a focus on improving the alliance, a cooperative stance, and effectively repairing ruptures [21]. Even more interestingly, assuring these generic features within a generalist treatment approach for PD turned out to be equally effective as specialist psychotherapies across various trials [19, 22-24].

Stressing the importance of basic generic principles above adherence to complex specialist psychotherapy models may have three additional advantages. Firstly, it could facilitate widespread dissemination of good-enough treatment, as training and supervision requirements may be reduced [25]. Secondly, some studies suggest that adherence to specialist programs is troublesome, negatively affecting outcome [26, 27]. Simplifying treatment principles may therefore improve ease of application and adherence. Finally, general principles may be applied more easily across different types of PD, while specialist theorydriven models are often focused more specifically on specific types, mostly Borderline PD.

These findings offer opportunities to provide better access to effective treatment for patients suffering from PDs. Instead of following the sometimes cumbersome and costly process of implementing evidence-based specialist programs, general quality of PD treatment may be more efficiently improved by enhancing the level of care in existing services in accordance with a common features approach [22]. The central issues then become how these common features can be organized within an integrative framework and what training is needed to assure their application. To address this issue, the Centre of Expertise on Personality Disorders (CEPD), a network organization for mental health institutes in the Netherlands, initiated the GIT-PD project in 2012. The aim was to make services more compliant to general principles of good care for PD patients and enhance uniformity across services in the Netherlands.

\section{Development of GIT-PD}

The development of the GIT-PD framework started with a review of specialist and generalist PD treatment manuals [22, 24] and assumed mechanisms of change [28]. Expert meetings were organized as focus groups to identify relevant features in the treatment of PDs. Specific attention was given to suitability for PD patients with severe mental illness given the experienced shortcomings of specialist treatments for this subgroup [10]. In a focus group of 15-20 professionals from 9 mental health care institutions, relevant principles and ingredients for effective care were discussed, based upon the available expertise and experience of participants. These principles were manualized and matched with outcomes of the literature search on general principles of good enough care for PDs, including upcoming generalist approaches like Structured Clinical Management [22] and Good Psychiatric Management [24]. This manual was presented in several rounds to the focus group until sufficient detail and consensus were obtained. Initial drafts of this manual were also presented and discussed with a representative group of PD patients and family members until consensus was reached. Both patients and family members stressed the importance of engaging relatives in all stages of treatment, which became one of the core 
components of the GIT-PD approach. As the manual is designed to allow a certain flexibility, new adaptations that were deemed necessary given developments in the field, could be introduced following the same process. This design reflected the 'nature' of the GIT-PD project: GIT-PD was designed top-down (using literature review) as well as bottom-up (using discussion in focus groups) in order to optimize an ongoing exchange of ideas by experts, professionals, patients and family members.

\section{Theoretical and clinical starting points}

Following the DSM-5 Alternative Model for PDs, GIT-PD assumes that all PD patients share impairments in personality functioning as reflected in Self and Interpersonal impairments [29]. Self-functioning refers to the experience of a unique identity and of a sense of self-direction. Interpersonal functioning refers to the capacity for empathy and intimacy. Specific features of GIT-PD are designed using this starting point. Firstly, PD patients' proneness to become emotionally dysregulated as part of their identityimpairments, may lead to repeating crises, impacting upon their environment, including family and professionals. This could generate iatrogenic responses such as overmedication or rapid hospitalization. Moreover, PD patients' impairment in self-direction may interfere with a stable commitment to change, motivation for treatment and ability to self-reflect to gain insight in their problems. Secondly, GIT-PD anticipates that empathy problems of PD patients will affect the therapist relationship and may interfere with patients' ability to learn socially from others, given their distrust and related suspiciousness towards the intentions of caregivers [30]. Their inability to tolerate intense treatment relationships may lead to alliance ruptures. It can also lead to lead to unhelpful emotional reactions in professionals or teams. The combined effect of the impairments that constitute the core of PD patients' problems, may interfere with the assumed working mechanisms of therapy, making any treatment less effective and, at times, even iatrogenic. GIT-PD focuses on securing principles that decrease the likelihood of these phenomena interfering with beneficial treatment processes.

\section{Core features of GIT-PD}

Firstly, GIT-PD is a framework. Compared to other approaches it is not primarily theory driven and prescriptive in terms of setting, modalities or content of sessions. GITPD allows institutions to keep local context-based traditions, while still improving their services. It assumes that the core effectiveness lies in a set of principles, rather than in specific content based on a strict adherence to any specific theoretical concept of personality. This enables not only a personalized approach based on a process of shared decision making with the patient to select the most suitable interventions, but also optimizes response to actual needs of the patients and families during treatment. Secondly, GIT-PD assumes that basic therapist skills - part of any training of mental health care professionals - can suffice to provide good-enough treatment, when applied with a professional stance adjusted to the interpersonal hypersensitivity of most PD patients. Thirdly, GIT-PD integrates the findings that the organizational context may have a large impact on treatment effectiveness [26]. Therefore, principles are formulated also at the level of contextual and organizational conditions, team effectiveness, therapist attitude and treatment focus.

\section{Outline of the GIT-PD treatment principles}

Table 1 summarizes the core features of the GIT-PD program. It contains the organizational requirements of the program, principles of the therapeutic process and shared common factors across treatments of PD patients, related to the core impairments of PD patients.

The table could be read bottom-up: the heart of GIT-PD is a common basic professional stance, characterized by active involvement, careful exploration, validating feedback mirroring the therapist's understanding of the patient's affects and honest, authentic and transparent discussion. Using this stance, therapists using GIT-PD will continuously focus upon common areas of vulnerability of PD patients: strengthening their identity by helping them focus upon their needs, affects, opinions and values; strengthening their sense of agency by encouraging the pursuit of goals and values, and by helping them understand their behavior; enhancing understanding of others and relationships; establishing secure, mutual intimate relationships, within and outside treatment, facilitating renewed social learning.

Therapists are always embedded in a team and some principles are formulated to enhance team effectiveness. This includes appointing a team member who monitors the process of the team discussion and enables a reflective stance and the integration of all perspectives on treatment progress. GIT-PD encourages shared caseload and active cooperation between team members. As a rule, interventions that deviate from usual practice are discussed with peer team members as a way to prevent unthoughtful action caused by strong emotional reactions.

Furthermore, interventions by team members should be oriented towards specific goals, which should be evaluated regularly. The treatment process should be structured in time, reflected by the clinical pathway of GITPD (see below).

At top level, managerial commitment and consistency over time are essential for implementing good care for PDs. Therefore, organizational prerequisites are addressed as a general principle as well. They refer to the contextual conditions, adequate managerial support, integration with other services to provide consistency in approach, and clear structure of the program. 
Table 1 General principles of GIT-PD

\begin{tabular}{|c|c|c|}
\hline Level & Principle & Explanation \\
\hline \multirow[t]{3}{*}{ Organizational } & Support & Sustained support of team by management board \\
\hline & Structure & $\begin{array}{l}\text { Predictability of program; e.g. schedule of appointments, availability of } \\
\text { team, exchange of information, responsibilities of team members. }\end{array}$ \\
\hline & Integration & Cooperation with other services, e.g. crisis and social services; within and between units \\
\hline \multirow[t]{2}{*}{ Treatment process } & Phased \& episodic & Structured in time (begin-middle-end) and possibly episodic to prevent relapses \\
\hline & Goal-focused & $\begin{array}{l}\text { cooperative agreement upon goals. Regular evaluation, leading to adaptations or } \\
\text { (premature) termination. }\end{array}$ \\
\hline \multirow[t]{3}{*}{ Team } & Complementary & Enabling integration of all perspectives in the team \\
\hline & Reflective & Enabling reflection upon team culture and processes \\
\hline & Supportive & Enabling mutual support to prevent iatrogenic actions \\
\hline \multirow[t]{4}{*}{ Common therapeutic factors } & $\begin{array}{l}\text { Self: Identity } \\
\text { (autonomy-focused) }\end{array}$ & $\begin{array}{l}\text { Strengthening autonomy; enhancing self-esteem; using strategies to identify, } \\
\text { regulate and cope with (intense) emotions, including crisis }\end{array}$ \\
\hline & $\begin{array}{l}\text { Self: Self-direction } \\
\text { (motivation-focused) }\end{array}$ & $\begin{array}{l}\text { Monitoring and fostering motivation to engage and change in treatment; establishing } \\
\text { strategies to improve self-reflection; Encouraging self-management of life stressors }\end{array}$ \\
\hline & $\begin{array}{l}\text { Interpersonal: Empathy } \\
\text { (other/context-focused) }\end{array}$ & $\begin{array}{l}\text { Monitoring, discussing and challenging patient's experiences of others, including the } \\
\text { therapist; focusing on understanding emotions and behavioral reactions of others }\end{array}$ \\
\hline & $\begin{array}{l}\text { Interpersonal: Intimacy } \\
\text { (relationship-focused) }\end{array}$ & $\begin{array}{l}\text { Enhancing trust in therapy; establishing an emotionally involved relationship; } \\
\text { repairing ruptures; involving relatives and restoring openness to social learning }\end{array}$ \\
\hline \multirow[t]{3}{*}{ Basic stance of therapists } & Curiously involved & Taking an investigative, curious, involved stance \\
\hline & Supportive, Empathic & Taking an empathic, validating and supportive stance \\
\hline & Transparent, authentic & Open to discuss actual events in the therapist relationship \\
\hline
\end{tabular}

In our opinion, GIT-PD principles are not specific for PDs alone and may also be considered as generic principles of good care. However, we believe that the specific impairments of people with PDs, like their emotional and behavioral dysregulation and their interpersonal distrust, may jeopardize the adherence of professionals and teams to these principles. Moreover, we believe that if professionals fail to adhere to these generic principles, PD treatment may be affected more than treatment of other conditions would be. Ultimately, PD treatment may even become iatrogenic [31].

\section{Clinical pathways of GIT-PD}

GIT-PD stresses that treatment should be structured in time. This includes that treatment should be phased and specific targets for each phase should be identified. Figure 1 depicts the clinical pathway.

In the first phase careful assessment takes place, followed by discussion of core problems and determining the focus of treatment by therapist and patient. Treatment planning should be a process of shared decision making, tailoring treatment to the needs of the individual patient, thus promoting commitment to treatment and motivation for change.

The second phase ("treatment phase") includes psychoeducation, crisis anticipation and execution of the selected treatment interventions. Ongoing attention to motivation and engagement is necessary. Review of treatment progress takes place on a regular basis. The third phase prepares for the termination of treatment. This phase includes anticipating possible relapsing symptoms, 'booster' sessions, usually in a lower frequency than in the treatment phase, and attention is being paid to recovery. Throughout all phases, the basic professional stance is maintained, focusing on collaboration and motivation.

\section{Supporting implementation by the Dutch Center of Expertise for personality disorders}

Local mental health care units are responsible for the implementation of the GIT-PD framework. The Center of Expertise for Personality Disorders (CEPD) has provided a range of tools to support implementation and maintenance:

\section{1) Online support and the GIT-PD website}

The website contains information on GIT-PD, including procedures for frequently occurring clinical issues, like crisis management, drop-out, and boundary transgressions. It also includes around 50 demonstrational videos, that provide exemplary interventions for mental health care workers. An online toolbox contains descriptions of specific modules, psycho-educational leaflets, implementation plans and more. These tools have been supplied by participating mental health care centers. This is part of the earlier mentioned process of promoting exchange of ideas and materials between mental healthcare facilities. 


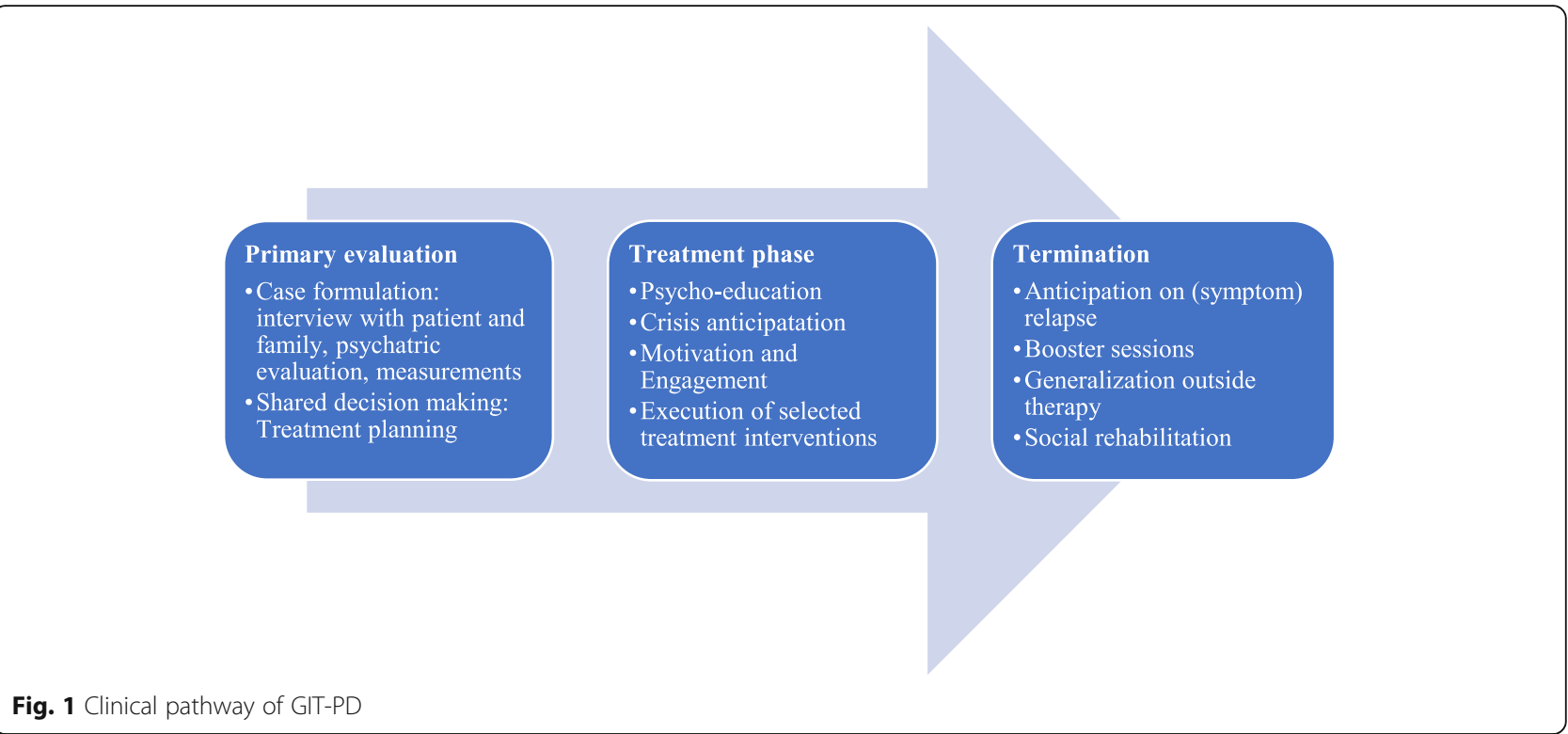

2) Training course

The CEPD provides a 2-day 'teach-the-teacher' training, which discusses the GIT-PD principles and offers active training on basic interventions, including the basic stance, assessment and treatment review, managing emotions and crises, and repairing ruptures. In addition, the training focuses on educational and supervising skills, enabling participants to subsequently train their own teams. Training materials are free to use for institutions participating in the CEPD. The course thus enables professionals of participating institutes to optimize the GIT-PD approach in their own teams.

\section{3) Template for managerial implementation}

A template for managerial implementation is available, which is based upon the current state-of-the-art literature about implementation of change in care. This outlines several steps for successful implementation, like making a diagnostic organizational analysis, mapping possible risks, interventions to tackle risks, and systematic planning of process monitoring. This template is offered as a support tool, but implementation itself is done by the local units.

\section{4) Quality maintenance}

Quality maintenance is provided through audits of participating services, organized and facilitated by the CEPD. Mental health care units are clustered into small groups. Professionals of mental healthcare units visit each other every 2 years and assess quality of care by using the GIT-PD principles as criteria.

\section{Discussion}

This paper discussed the GIT-PD initiative in the Netherlands. Essentially, GIT-PD strips down the complexities of specialist evidence-based treatment approaches to keep the most accessible and valuable basic principles of good-enough care for PD patients. The GIT-PD framework delivers practicable principles that can guide management of PD patients at the level of the therapist, the team and the organization. This framework is designed to provide a framework that is easy to implement and viable to adhere to over a longer time, helping to provide good-enough treatment for a broad group of patients with PDs. It was designed as an alternative for the prevailing treatment as usual given the often-insufficient capacity of specialist evidence-based programs. Additionally, as the GIT-PD principles are addressing the core impairments of PDs, it may provide an evidence-informed alternative for the treatment of the broad range of PDs. Ultimately, we believe both specialist and generalist approaches should play their role in the treatment of PDs, either in a stepped or matched care approach. Since the introduction of GIT-PD, there is tendency shift towards specialist psychotherapeutic centers in the Netherlands using GIT-PD as a basic treatment program for the majority of their patients, while reserving their specialist (MBT, DBT and SFT) programs for more complex patients who often have had an extended history of treatment failures.

\section{Key factors in the successful reform of PD services}

The GIT-PD project was initiated in 2012, starting with 8 mental health care centers. Now, 8 years from the start, 20 large Dutch health care institutions participate, and they 
have restructured their PD treatment units according to the GIT-PD principles. This reform has made psychotherapeutically informed treatment available to a larger and broader range of PD patients across the country.

We believe several factors have contributed to this success. GIT-PD was partly designed 'bottom-up' and professionals of all participating institutions and patients have been closely involved, also in the implementation phase across the institutes. GIT-PD is not strongly theory driven nor is it prescriptive allowing institutions to follow local preferences and methods.

Training therapists in specialist psychotherapies is a costly and long-term investment that often runs into budgetary constraints. GIT-PD offers an easier and cheaper way to provide basic skills for the treatment of PD patients. Moreover, training programs for specialist psychotherapies are reserved for registered psychotherapists (psychologists/psychiatrists), while most professionals in the field are social workers or nurses. The upgrading of existing services for PD has highly supported professionals and gave them an increased sense of agency in their work with this difficult-to-treat patient group.

Finally, although specialist treatment programs in the Netherlands now tend to include more severely impaired PD patients with a history of previously failed treatments, still the most severe mentally ill PD patients are excluded from these programs, as they suffer from too many crises, disrupting social problems or because they lack basic psychological mindedness. Consequently, treatments for these severely ill patients often lack any structure and methodological coherence. Due to a flexible delivery of interventions, the team approach and contextual support, GIT-PD provides a better alternative for these patients than the existing services.

\section{Similar initiatives}

Given the high societal costs associated with mental disorders, the improvement of access to effective psychological treatment is a major challenge for mental health care world-wide. In England, the 'Improving Access to Psychological Therapies' (IAPT) initiative by the National Health Service (NHS) aims to make evidence-based treatment for anxiety and depressive disorders available to a larger number of patients [32]. In Australia, the Project Air Strategy for Personality disorders is a partnership between the University of Wollongong and the NSW Ministry for Health, aiming to support better treatments for personality disorders [33]. What both projects have in common with GIT-PD are a dissemination strategy, training opportunities, and supervision of participating therapists. It should be noted however, that IAPT aims at improving treatment outcome for anxiety disorders and depression by promoting specific types of evidence-based treatment, linked to scientific evaluation from the start. Project Air and GIT-PD have both been developed to provide better evidenceinformed care by encouraging cooperation among services, professionals, families and patients. The focus in both projects so far is on the organizational requirements that services need to deliver beneficial and sustainable treatment programs for patients with PD. However, unlike Project Air, the GIT-PD project was initiated by the local mental health institutions and is also fully financed by these services without additional funding.

\section{Limitations}

The aim of the GIT-PD project is to improve treatment as usual by identifying common features of evidencebased treatment approaches and by implementing pragmatic principles related to good care for PDs. Although inter-institutional audits are conducted regularly to improve quality, data are not collected in a way that allows the monitoring of the impact on adherence and outcomes in clinical practice. Therefore, the GIT-PD project lacks scientific evaluation and evidence of the effectiveness in clinical practice is not yet available. As described, we believe the success of the GIT-PD lies in its flexibility and its personalized approach for patients and their relatives. However, this also generates a heterogeneity that may complicate systematic evaluation in commonly applied research designs. Nevertheless, we believe future studies could use a template GIT-PD program to study its effectiveness compared to specialist psychotherapy programs. Alternatively, aggregated data from routine outcome monitoring could be used if institutions are willing to harmonize data collection protocols.

\section{Conclusion}

GIT-PD has become a widespread and well-known standard treatment approach in the PD field in the Netherlands. Almost all major mental health centers participate in the project on a voluntarily basis forming a network structure supported by the national CEPD. We hope the GIT-PD project can serve as an attainable and cost-effective template for other countries as well in order to improve the general quality of care for the large group of patients suffering from severe personality pathology. ${ }^{1}$

${ }^{1}$ People who may be interested in some of the (subtitled and translated) materials can contact the first (J.H.) or second (E.W.) author. 


\section{Acknowledgments}

NA

\section{Authors' contributions}

$\mathrm{JH}$ designed and wrote the first draft, NB, EW and RV edited and added from their area of expertise. All authors agree with the final version. The author(s) read and approved the final manuscript.

\section{Funding}

NA

\section{Availability of data and materials}

NA

\section{Ethics approval and consent to participate}

NA

\section{Consent for publication}

NA

\section{Competing interests}

NA

\section{Author details}

Viersprong Institute for Studies on Personality Disorders, Halsteren, The Netherlands. ${ }^{2}$ Centre of Expertise on Personality Disorders, Utrecht, The Netherlands. ${ }^{3}$ Parnassia Psychomedical centre, The Hague, The Netherlands. ${ }^{4} \mathrm{GGZ}$ Oost Brabant, Boekel, The Netherlands. ${ }^{5}$ Rino Zuid, Eindhoven, The Netherlands. ${ }^{6}$ Tilburg University, Tilburg, The Netherlands. ${ }^{7}$ Arkin Mental Health Centre, Amsterdam, The Netherlands.

\section{Received: 30 January 2020 Accepted: 9 July 2020}

Published online: 10 August 2020

\section{References}

1. Grant BF, Hasin DS, Stinson FS, Dawson DA, Chou SP, Ruan WJ, Pickering RP. Prevalence, correlates, and disability of personality disorders in the United States: results from the National Epidemiologic Survey on alcohol and related conditions. J Clin Psychiatry. 2004;65(7):948-58.

2. Zanarini MC, Frankenburg FR, Reich DB, Fitzmaurice G. Attainment and stability of sustained symptomatic remission and recovery among patients with borderline personality disorder and axis II comparison subjects: a 16year prospective follow-up study. Am J Psychiatr. 2012;169(5):476-83.

3. Fok MLY, Hayes RD, Chang CK, Steward R, Callard FJ, Moran P. Life expectancy at birth and all-cause mortality among people with personality disorder. J Psychosom Res. 2012;73(2):104-7.

4. Soeteman DI, Verheul R, Delimon J, Meerman AM, Van den Eijnden E, Rossum BV, Kim JJ. Cost-effectiveness of psychotherapy for cluster B personality disorders. Br J Psychiatry. 2010;196(5):396-403.

5. Stoffers-Winterling JM, Völlm BA, Rücker G, Timmer A, Huband N, Lieb K. Psychological therapies for people with borderline personality disorder. Cochrane Database Syst Rev. 2012:15(8):252.

6. Landelijke Stuurgroep Multidisciplinaire Richtlijnontwikkeling in de GGZ. Multidisciplinaire richtlijn persoonlijkheidsstoornissen. In: Richtlijn voor de diagnostiek en behandeling van volwassen patiënten met een persoonlijkheidsstoornis. Utrecht: Trimbos-instituut; 2008.

7. National Collaborating Centre for Mental Health. Borderline personality disorder: treatment and management. Leicester: British Psychological Society; 2009.

8. National Health and Medical Research Council. Clinical practice guideline for the management of borderline personality disorder. Melbourne; 2012. National Health and Medical Research Council.

9. Fok MLY, Stewart R, Hayes RD, Moran P. The impact of co-morbid personality disorder on use of psychiatric services and involuntary hospitalization in people with severe mental illness. Soc Psychiatry Psychiatr Epidemiol. 2014;49(10):1631-40.

10. Hermens ML, Van Splunteren PT, Van den Bosch A, Verheul R. Barriers to implementing the clinical guideline on borderline personality disorder in the Netherlands. Psychiatr Serv. 2011:62(11):1381-3.

11. Iliakis EA, Sonley AKI, Ilagan GS, Choi-Kain LW. Treatment of borderline personality disorder: is supply adequate to meet public health needs? Psychiatr Serv. 2019;70(9):772-81. https://doi.org/10.1176/appi.ps.201900073.
12. Bales DL, Verheul R, Hutsebaut J. Barriers and facilitators to the implementation of mentalization-based treatment (MBT) for borderline personality disorder: key factors in the implementation of mentalizationbased treatment (MBT). Personal Ment Health. 2017b;11(2):118-31.

13. King, J.C., Hibbs, R., Saville, C.W.N., \& Swales, M.A. (2018). The survivability of dialectical behaviour therapy programmes: a mixed methods analysis of barriers and facilitators to implementation within UK healthcare settings. BMC Psychiatry, 18: 302. https://doi.org/https://doi.org/10.1186/s12888-0181876-7.

14. Swales MA, Taylor B, Hibbs RA. Implementing dialectical behaviour therapy: Programme survival in routine healthcare settings. J Ment Health. 2012; 21(6):548-55.

15. Zanarini MC, Frankenburg FR, Reich DB, Conkey LC, Fitzmaurice GM Treatment rates for patients with borderline personality disorder and other personality disorders: a 16-year study. Psychiatr Serv. 2014;66(1):15-20.

16. Olesen J, Gustavsson A, Svensson M, Wittchen HU, Jönsson B. The economic cost of brain disorders in Europe. Eur J Neurol. 2012:19(1):155-62.

17. Bamelis LL, Arntz A, Wetzelaer P, Verdoorn R, Evers SM. Economic evaluation of schema therapy and clarification-oriented psychotherapy for personality disorders: a multicenter, randomized controlled trial. J Clin Psychiatry. 2015; 76(11):1432-40.

18. Bateman A, Fonagy P. Treatment of borderline personality disorder with psychoanalytically oriented partial hospitalization: an 18-month follow-up. Am J Psychiatr. 2001;158(1):36-42.

19. Christea IA, Gentili C, Cotet CD, Palomba D, Barbui C, Cuijpers P. Efficacy of psychotherapies for borderline personality disorder: a systematic review and meta-analysis. JAMA Psychiatry. 2017;74(4):319-28.

20. Weinberg I, Ronningstam E, Goldblatt MJ, Schechter M, Maltsberger J. Common factors in empirically supported treatments for borderline personality disorder. Curr Psychiatry Rep. 2011;13(1):60-8.

21. Norcross JC, Lambert MJ. Psychotherapy relationships that work III. Psychotherapy. 2018:55(4):303-15.

22. Bateman AW, Krawitz R. Borderline personality disorder: an evidence-based guide for generalist mental health professionals. Oxford: Oxford University Press; 2013.

23. Chanen AM, Jackson HJ, McCutcheon LK, Jovev M, Dudgeon P, Yuen HP, McGorry PD. Early intervention for adolescents with borderline personality disorder using cognitive analytic therapy: randomised controlled trial. $\mathrm{Br}$ J Psychiatry. 2008;193(6):477-84.

24. Gunderson JG, Links P. Handbook of good psychiatric management for borderline personality disorder. Arlington: American Psychiatric Publishing; 2014.

25. Choi-Kain LW, Albert EB, Gunderson JG. Evidence-based treatments for borderline personality disorder: implementation, integration, and steppedcare. Harv Rev Psychiatry. 2016;25(5):342-56.

26. Bales DL, Timman R, Luyten P, Busschbach J, Verheul R, Hutsebaut J. Implementation of evidence-based treatments for borderline personality disorder: the impact of organizational changes on treatment outcome of mentalization-based treatment. Personal Ment Health. 2017a;11(4):266-77.

27. Norrie J, Davidson K, Tata P, Gumley A. Influence of therapist competence and quantity of cognitive behavioural therapy on suicidal behaviour and inpatient hospitalisation in a randomised controlled trial in borderline personality disorder: further analyses of treatment effects in the BOSCOT study. Psychol Psychother: Theory Res Pract. 2013;86(3):280-93.

28. Castonguay LG, Beutler LE. Principles of therapeutic change that work. Oxford: Oxford University Press; 2006.

29. Bender DS, Morey LC, Skodol AE. Toward a model for assessing level of personality functioning in DSM-5, part I: a review of theory and methods. J Pers Assess. 2011;93(4):333-46.

30. Fonagy $\mathrm{P}$, Luyten $\mathrm{P}$, Allison $\mathrm{E}$. Epistemic petrification and the restoration of epistemic trust: a new conceptualization of borderline personality disorder and its psychosocial treatment. J Personal Disord. 2015:29(5):575-609.

31. Fonagy $\mathrm{P}$, Bateman AW. Progress in the treatment of borderline personality Disdorder. Br J Psychiatry. 2006;188(1):1-3.

32. Clark DM. Realizing the mass public benefit of evidence-based psychological therapies: the IAPT program. Annu Rev Clin Psychol. 2018;14:159-83.

33. Grenyer BFS. An integrative relational step-down model of care: the project air strategy for personality disorders. Aust Clin Psychol Assoc. 2014;9:8-13.

\section{Publisher's Note}

Springer Nature remains neutral with regard to jurisdictional claims in published maps and institutional affiliations. 